\title{
Hospital care in Departments defined as COVID-free: A proposal for a safe hospitalization protecting healthcare professionals and patients not affected by COVID-19
}

\author{
Rosario Leonardi ${ }^{1}$, Piera Bellinzoni ${ }^{2}$, Luigi Broglia ${ }^{2}$, Renzo Colombo ${ }^{3}$, Davide De Marchi ${ }^{2}$, \\ Lorenzo Falcone ${ }^{1}$, Guido Giusti ${ }^{2}$, Vincenzo Grasso ${ }^{1}$, Guglielmo Mantica ${ }^{4}$, Giovanni Passaretti ${ }^{2}$, \\ Silvia Proietti ${ }^{2}$, Antonio Russo ${ }^{2}$, Giuseppe Saitta ${ }^{2}$, Salvatore Smelzo ${ }^{2}$, Nazareno Suardi ${ }^{4}$, \\ Franco Gaboardi ${ }^{2}$, UrOP Executive Committee * \\ ${ }^{1}$ Musumeci GECAS Clinic, Gravina di Catania, Catania, Italy; \\ ${ }^{2}$ Department of Urology, San Raffaele Turro Hospital, Milan, Italy; \\ ${ }^{3}$ Department of Urology, San Raffaele Hospital, Milan, Italy; \\ ${ }^{4}$ Department of Urology, IRCCS Policlinico San Martino Hospital, University of Genova, Genova, Italy;
}

\begin{abstract}
Summary The COVID-19 pandemic influenced the normal course of clinical practice leading to significant delays in the delivery of healthcare services for patients non affected by COVID-19. In the near future, it will be crucial to identify facilities capable of providing health care in compliance with the safety of healthcare professionals, administrative staff and patients. All the staff involved in the project of a Covid-free hospital should be subjected to a diagnostic swab for COVID-19 before the beginning of healthcare activity and then periodically in order to avoid the risk of contamination of patients during the process of care. The modifications of various activities involved in the process of care are described: outpatient care, reception of inpatients, inpatient ward and operating room. For outpatient care, modality of appointment procedure, characteristics of waiting room and personal protective equipment (PPE) for healthcare professionals and administrative staff are presented. Reception of inpatients shall be conditional on a negative swab for COVID-19 obtained with a drive-in procedure. The management of the operating room represents the most crucial step of the patient's care process. The surgical team should be restricted and monitored with periodic swabs; surgical procedures should be performed by experienced surgeons according to standard procedures; surgical training experimental treatments and research protocols should be suspended. Adequate personal protective equipment and measures to reduce aerosolization in the operating room (closed circuits, continuous cycle insufflators, fume extraction) should be adopted. Prevention of possible transmission of the virus during procedures in open, laparoscopic and endoscopic surgery is to use a multi-tactic approach, which includes correct filtration and ventilation of the operating room, the use of appropriate PPE (FFP3 plus surgical mask and protective visor for all the staff working in the operating room) and smoke evacuation devices with a suction and filter system.
\end{abstract}

KEY WORDS: COVID-19; Pandemy; Surgery; Endoscopy; Filtration.

Submitted 20 April 2020; Accepted 21 April 2020

\section{INTRODUCTION}

This document was based on the review of information materials from AGENAS (Agenzia Nazionale per i Servizi Sanitari), SIU (Italian Society of Urology), SAGES (American
Society of Gastroenterology Endoscopic Surgery), EAES (European Society of Endoscopic Surgery) and Italian Society of Endoscopic Surgery. Prevention measures, personal protective equipment (PPE) and protocols for healthcare professional, administrative staff and patients have been included trying to implement the best prevention measures against COVID-19 infection in public or private healthcare facilities.

The COVID-19 pandemic influenced the normal course of clinical practice through multiple mechanisms leading to significant delays in the delivery of healthcare services. The possibility of meeting the demand for healthcare in the near future will depend on the duration of the epidemic, its economic and social consequences, and also on changes of the population caused by the infection itself. In consideration of the awareness that for many months we will still have to live with the presence of the virus among the population, it becomes mandatory an immediate rationalization of resources in order to ensure continuity of healthcare for patients not affected by COVID-19. It is crucial to identify facilities capable of providing health care in compliance with the safety of healthcare professionals, administrative staff and patients who need medical treatment. The triage of medical and surgical procedures must take into account the heterogeneity of the pathologies to be treated, the variability of the timing useful for effective treatment, the different surgical approaches and non-surgical alternatives. It should not be forgotten that the epidemic has heterogeneous loco-regional outbreaks with differentiated measures from local government authorities.

Although there is no official data, it has been reported that a new health migration is underway, with a flow of patients moving from high endemic areas to areas where they can obtain adequate care (AGENAS-SIU).

This document aims to provide indications in the triage of patients and in the optimization of the resources available in this difficult moment. As a precondition, all the staff involved in the project of a Covid-free hospital should be subjected to a diagnostic swab for COVID-19 before the beginning of healthcare activity. The swab should be

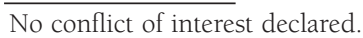


repeated every two weeks in order to avoid the risk of contamination of patients during the process of care. If reliable tests will be validated, able to replace the swab in the diagnosis of COVID-19, these tests could be considered as faster and less expensive screening procedure to monitor the absence of infection in the medical staff and hospital nursing.

We divided the text into several chapters concerning the various activities involved in the process of care:

- Outpatient care

- Reception of inpatients

- Inpatient ward

- Operating room.

\section{Outpatient visits}

\section{Appointment procedure}

The outpatient visit should be booked through a telephone triage.

\section{Exclusion criteria:}

- Coming from high endemic areas (red areas)

- Referred flu symptoms (sore throat, cough, rhinorrhea)

- Temperature

- State of quarantine or cohabitation with subjects in compulsory quarantine.

Patients must be informed that they must access to the office equipped with a surgical mask.

The patient can be accompanied by a person who however will not enter in the office at the time of the visit. If it is necessary to provide information to the companion, this will be convened separately so that the criteria for social distancing are respected within the office.

Upon entering in the facility it would be useful to perform a body temperature measurement.

\section{Waiting room}

In relation to the size of the waiting room, one or more patients can be accepted.

It is mandatory to maintain a distance between the patients and their companions of at least two meters.

Healthcare professionals

Healthcare professionals must be equipped with Personal Protective Equipment (PPE) including FFP3 mask and protective goggles or visors.

Administrative staff (payment of outpatient service) Must be equipped with PPE (FFP2 mask).

Note: It is important that the area of the consultations must be separated from the area of hospitalization.

\section{Hospitalization of the patient for surgery}

Indispensable condition for taking care of the patient (swab testing)

An indispensable condition for the patient to be candidate for surgical treatment is that of being subjected to a swab for COVID-19 before entering the facility.

How and how long before admission, it depends on the structural and organizational characteristics of the hospital and the time needed to obtain the result of the swab.
Our suggested option is the drive-in swab. A protected area (parking) must be identified where the car with the patient on board can stop for the time necessary to perform the swab. The staff responsible for the procedure must be provided with the maximum individual protection (suit, FFP3 mask, protective visor and gloves).

In the case that the Hospital could arrange a special area for pre-hospitalization the patient could be accepted after the swab in this area of the hospital to wait for the response.

\section{General criteria}

Protect the patient from potential Covid infections in the hospital setting.

Protect staff and other patients from contamination, including mutual.

The surgical team should be restricted and monitored with periodic swabs; surgical procedures should be performed by experienced surgeons according to standard procedures; surgical training experimental treatments and research protocols should be suspended.

Admission of the patient to the hospital for elective surgery The call of patients for elective surgery must take place through a telephone interview to rule out a possible contagion from Coronavirus SARS-CoV-2:

- clinical criteria: cough, rhinorrhea, body temperature rise, pharyngodynia, abdominal pain, conjunctivitis

- epidemiological criteria: direct contact with positive COVID-19 patient (cohabitation/interview for more than 15 minutes in the same environment, attendance at hospital facilities); origin from high endemic geographic areas identified by current epidemiological data

The patient must be informed that the following steps will be followed on the day of the call to enter the hospital:

- arrival at the facility will take place starting at 7.30 with a staggering between one patient and the other of about $15 \mathrm{~min}$

- arrival by car and parking in the drive-in swab area.

- return to his/her home where the patient must respect strict self-isolation until he/she receives the call from the triage staff who communicates the outcome of the swab

- as an alternative (especially for patients domiciled far away from the hospital), the hospital could offer hospitality in a separate area with hotel service in individual rooms with private bathroom (nursing and service staff of this area must wear suitable personal protective equipment including gloves, FFP2 mask, plus surgical masks, if the former are equipped with an exhalation valve, waterproof gown for contacts, and protective visor)

- any kind of personal contact of the patient with the other patients has to be avoided

- in the case of a delayed result of the swab, food must be delivered out of the room in disposable tray with a sealable bag where the patient should pour food waste including the tray

- food waste must be handled as special hazardous waste

- room must undergo sanitization at the end of the stay

- in the event of a negative outcome of the swab, the patient can be admitted to the ward, staying fasting, to start the acceptance and hospitalization procedure 
- in the event of a positive outcome, the patient is invited to inform the competent health authorities to arrange for self-isolation at home or hospitalization in the Covid ward.

The patient can be accompanied by a family member or friend who will be able to assist him/her in the swab procedure and in the subsequent phase of hospitalization but who will not be admitted to the wards.

Both the patient and the companion, in the various stages of the swab procedure and hospitalization must be equipped with a mask.

\section{Entry of the patient to the facility}

\section{Waiting room}

Waiting room must be organized taking into account social distancing.

\section{Administrative staff appointed to accept the patient} Front-office administrative staff must carry out their activity protected by a transparent glass barrier and equipped with PPE (FFP2 mask).

Medical and nursing staff appointed to accept the patient Collection of clinical history, compilation of the clinical records and administration of the informed consent must be done with compliance of the distancing measures.

Discussion of consent to surgery should include an illustration of the risk of COVID-19 exposure and its potential consequences on clinical outcomes.

Personnel dedicated to blood sampling for blood chemistry and to cardiological and anesthesiological evaluation must be equipped with PPE (FFP2 masks with or without surgical mask based on the presence or absence of an exhalation valve, protective visor, gloves and waterproof gown). Everything can take place in a dedicated area where the rules of social distancing are respected or directly in the hospital room.

Note: The attending physician and/or the patient's reference specialist should be invited to inform the head of the anesthesia department or his/her delegate, before the hospitalization phase, if there are, in his/her opinion, pathologies that may contraindicate the intervention or that could request diagnostic investigations before hospitalization. In this case, the patient should be booked for an anesthesiological outpatient visit in a reasonable time before admission.

\section{Entry of the patient into the ward}

After the acceptance phase and the first phase of evaluation in preparation for the surgical procedure, the patient is sent to the hospital ward and housed in a single room with personal bathroom. The medical and nursing staff, in this phase, must always wear suitable personal protective equipment (gloves, FFP2 mask, plus surgical masks, if the former are equipped with an exhalation valve, waterproof gown for contacts, protective visor).

\section{Management of the operating room}

\section{General considerations}

Management of the operating room represents the most crucial step of the patient's care process. Even if the patient underwent a swab the day before with a negative result, this is not sufficient to safely exclude a positivization in the following day.

In the operating room for a whole series of conditions related to intubation and to the use of endoscopic and laparoscopic surgical instruments, the risk of transmission is maximum. For this reason, the most effective PPE systems must be adopted and it is necessary to implement well-coded behaviors that can be summarized as follows:

- the surgical team should be restricted, monitored with periodic swabs and subjected to restrictive measures to limit the risk of infection

- there should be no change of staff in the room

- surgical procedures should be performed by experienced surgeons to reduce operating times and the risk of complications (therefore, the suspension of surgical training is recommended

- the standard approach should be maintained, in order not to compromise the outcome and standardize the operating room times

- surgical staff should not stay in the operating room during intubation maneuvers, waiting a few minutes from their conclusion before entering, leaving any infected droplets to settle

- it would be desirable for intubation and extubation to take place inside a negative pressure room

(https://www.asahq.org/in-the-spotlight/coronavirusCOVID-19-information) $(1,2)$

- all measures should be put in place to reduce the risk of contagion among healthcare professionals by adopting adequate personal protective equipment. All members of the operating room staff must use PPE (FFP3 mask). Appropriate clothing and face shields must be used. These measures should be used during all pandemic surgical procedures, regardless of known or suspected Covid status. The positioning and removal of PPE must be performed according to the World Health Organization (WHO) and Centre for Disease Control and Prevention (CDC) guidelines (https://www.cdc.gov)

- measures to reduce aerosolization in the operating room should be considered (closed circuits, insufflators continuous cycle, fume extraction).

Note: Remember that the virus has been isolated very frequently in respiratory secretions, saliva and feces, rarely in the blood, exceptionally in the urine.

\section{Specific technical considerations}

There is very little evidence regarding the risks of virus spread related to the use of the laparoscopic surgical technique compared to the open approach (3). Emerging evidence and regular updates are provided by the website https://siceitalia.com/covid19/

It is recommended, however, to seriously consider the possibility of viral contamination of operating room personnel during surgery, be it open, laparoscopic or robotic. It is advisable to actively monitor strict application of protective measures for the safety of the operating room staff. Although previous research has shown that the laparoscopic technique can favor the aerosolization of pathogens present in the blood (Corynebacteria, Papillomavirus and HIV) (4-6), there is no evidence to indicate that this effect 
is also possible for the coronavirus, nor that the risk can be confined exclusively to minimally invasive surgical procedures. However, as a precaution, coronavirus should potentially be considered capable of aerosolizing. For this reason, the use of devices to filter $\mathrm{CO}_{2}$ should be strongly considered.

For further information https://eaes.eu/eaesand-sages-recommendations-regarding-surgical-response-to-covid-19-crisis/ The proven benefits of minimally invasive treatment in terms of reduced duration of hospitalization and reduction of complications, as well as the potential advantages in terms of ultrafiltration of most or all aerosol particles, must be strongly considered. In fact, the filtration of aerosolized particles can be more difficult during open surgery.

There may be an increased risk of virus exposure for endoscopists during upper gastrointestinal tract and airway procedures. When these procedures are necessary, the rigorous use of PPE of greater protection is recommended. The complete mask with FFP 3 should be considered for the whole team, following the guidelines of CDC (https://www.cdc.gov) or WHO (https://www. who.int) $(5,6)$.

\section{Filtration}

The smaller droplets, in contact with the ambient air (aerosol) can transmit the infection from one individual to another via the respiratory tract (within a certain distance). Currently, the "droplet" diameter classification system (from 5 to $10 \mu \mathrm{m}$ ) represents the unit of measurement used to evaluate the transmission mode of an infectious disease. Filtration can be an effective means of protection from virus release during minimally invasive surgery and endoscopy. Masks such as N95 respirators are designed to filter $95 \%$ of 0.3 micron and larger particles. Respiratory masks of protection class FFP3 offer the maximum possible protection from the pollution of breathing air with a protection of at least $99 \%$ from particles up to $0.6 \mu \mathrm{m}$ in size.

Purified air respirators (PAPR) can be useful for intubation, extubation, bronchoscopy, endoscopy and tracheostomy. Intraoperatively, filters are used to remove smoke and particles including viruses. Air particulate filters (HEPA) have a minimum efficiency index of $99.97 \%$ for the removal of particles with a diameter greater than or equal to 0.3 microns (6).

ULPA (Ultra-Low Particulate Air) filters can remove 99.999\% of airborne particles with a minimum particle penetration size of 0.05 microns.

The Association of periOperative Registered Nurses (AORN) guidelines define ULPA as filters capable of removing particles of 0.1 microns (7). Filtration is also essential on a large scale in positive pressure operating rooms. HEPA filters positioned in the ceiling provide terminal cleaning.

Currently, the best practice to reduce the possible transmission of the virus during procedures in open, laparoscopic and endoscopic surgery is to use a multi-tactic approach, which includes correct filtration and ventilation of the operating room, the use of appropriate PPE (FFP3 plus surgical mask, plus protective visor for all staff working in the operating room) and smoke evacuation devices with a suction and filter system (8).
Practical filtration measures during laparoscopic surgery

1. The pneumoperitoneum must always be safely evacuated from the trocar connected to the filtration device before removing the trocars, extracting the operating piece or converting by laparotomy.

2. Once the trocars are positioned, their valves should not be opened during surgery, if possible. If it is necessary to change the insufflation site on another trocar, the valve must be closed before disconnecting the tubing and the valve of the new trocar must remain closed until the insufflation tubing has been connected. The insufflator must be activated before the new insufflation valve is open. This is to prevent the backflow of gas into the insufflator itself.

3. During the desufflation phase, all gas and exhaust fumes must pass through an ultra-filtration system, possibly activating the desufflation mode on the insufflator, if this mode is available.

4. If the insufflator in use does not have a desufflation function, it must be ensured that the valve that is used for insufflation has been closed before the $\mathrm{CO}_{2}$ flow is deactivated (even if there is a filter in line with the tube). Without taking this precaution, contaminated intra-abdominal $\mathrm{CO}_{2}$ can be pushed into the insufflator when the intra-abdominal pressure is higher than the pressure inside the insufflator.

5. The patient must be on the level at the time of desufflation.

6. The workpiece(s) must be extracted once all the $\mathrm{CO}_{2}$ gas and smoke have been evacuated.

7. Drainage pipes should only be used if absolutely necessary.

8. The methods of closing the accesses of the trocars with the use of sutures that allow the escape of gas and residual fumes must be avoided. The fascial plane must be closed after complete desufflation.

9. Laparoscopic hand-assisted techniques can lead to significant $\mathrm{CO}_{2}$ and smoke losses and should therefore be avoided. A protection system can be positioned after complete desufflation to remove more voluminous operating pieces and protect the wound. The piece can then be removed and closed.

Systems for the evacuation of smoke and gas

Below is a list of commercially available products that could potentially be used to filter $\mathrm{CO}_{2}$ gas or smoke evacuated during surgical procedures (Table 1 ).

The list was provided by SAGES and EAES that stated they do not promote any of the following products. SAGES and EAES specify that they have sought information by contacting the known manufacturers, but the possibility is recognized that there are many other companies that may have similar products on sale. Surgeons should be aware of the characteristics of the products used in their facility and contact the product representative or refer to the product instructions for their use.

For a consultation of the smoke and gas evacuation systems currently on the market, refer to the links:

https://www.sages.org/resources-smoke-gas-evacuation-duringopen-laparoscopic-endoscopicprocedures/https://www.sages. org/wp-content/uploads/2020/03/Summary-of-CommerciallyAvailablePneumoperitoneum-Smoke-Evacuation-Systems.pdf 
Table 1.

Commercially available systems for the evacuation of smoke and gas.

\begin{tabular}{|c|c|c|c|c|c|c|c|}
\hline Company & ConMed & Cooper & Ethicon & Medtronic & Olympus & Stryker & Northgate \\
\hline \multirow[t]{4}{*}{ Commercial brand } & AirSeal $^{\oplus}$ (lap) & SeeClear ${ }^{\circledR}$ Plume-Away & Megadyne & ValleyLab RapidVac'TM & UHI-4 & Pneumoclear ${ }^{T M}$ & Nebulae ${ }^{\mathrm{TM}}$ I \\
\hline & PlumePen $^{\circledast}$ (open) & & Mega Vac Plus & & & PureView ${ }^{T M}$ & \\
\hline & Buffalo Filter ${ }^{\circledast}$ & & Mega Vac ${ }^{\mathrm{TM}}$ & & & & \\
\hline & Smoke Management & & Mini Vac ${ }^{\mathrm{TM}}$ & & & & \\
\hline Open & Yes & No & Yes & Yes & No & No & No \\
\hline
\end{tabular}

In addition to the smoke evacuation products, the Ultravision system can minimize aerosolized particles inside the pneumoperitoneum (https://www.sages.org/ wpcontent/uploads/2020/03/Ultravision-as-an-adjunct. pdf).

\section{Electrosurgical and laser units}

The electrosurgical units and lasers must be programmed to the lowest possible settings for the desired effect and for the correct execution of the surgery.

The use of monopolar electrosurgery, ultrasound dissectors and advanced bipolar devices and lasers can lead to aerosolization of the particles. If available, the use of monopolar electrosurgical units with integrated smoke aspirator is recommended (9-15).

During their use, the rules of maximum protection of the medical nursing staff apply as required for all procedures in the operating room.

Measures to be put into practice during laparoscopic surgery

1. The skin incisions should be as small as possible to allow trocars to pass and at the same time prevent $\mathrm{CO}_{2}$ losses around the trocars.

2. The $\mathrm{CO}_{2}$ insufflation pressure should be kept to a minimum and, if available, ultra-filtration (smoke evacuation or filtration system) should be used (https://www.sages.org/resources-smoke-gas-evacuationduring-open-laparoscopic-endoscopicprocedures/).

3. All pneumoperitoneum must be safely evacuated through a filtration system before removal of the trocars, extraction of the surgical piece, or conversion by open surgical procedure.

Measures to be put into practice during digestive endoscopy procedures

(https://www. asge.org/home/joint-gi-society-message-covid19) $(16,17)$.

1. In the absence of the ability to control the aerosolized virus during endoscopic procedures, all members of the endoscopy room or operating room must wear appropriate PPE (FFP3 mask, appropriate clothing and face shields). The positioning and removal of PPE must be carried out according to the CDC guidelines (https://www.cdc.gov).

2. Since patients can present with gastrointestinal manifestations of COVID-19, all endoscopic procedures in an emergency regime should be considered high risk.

3. Since the virus has been found in multiple cells of the gastrointestinal tract and in all fluids (saliva, enteric content, feces and blood) surgical energy must be minimized.
4. Endoscopic procedures that require additional insufflation of $\mathrm{CO}_{2}$ or ambient air should be avoided until we have a better understanding of the aerosolization properties of the virus. This includes many of the endoscopic mucosal resection procedures and endoluminal procedures.

5. Removing the caps on the endoscopes could release fluid and/or air and should be avoided.

\section{REFERENCES}

1. Wax RS, Christian MD. Practical recommendations for critical care and anesthesiology teams caring for novel coronavirus (2019$n \mathrm{CoV})$ patients. Can J Anaesth. 2020 Feb 12 [Epub ahead of print].

2. Zucco L, Levy N, Ketchandji D, et al. Anesthesia Patient Safety Foundation. https://www.apsf.org/news-updates/perioperativeconsiderations-for-the-2019-novel-coronavirus-covid-19/. Visited the $14^{\text {th }}$ April 2020.

3. Zheng MH, Boni L, Fingerhut A. Minimally invasive surgery and the novel coronavirus outbreak: lessons learned from Italy. Annals of Surgery. 2020. [Accepted for Publication].

4. Alp E, Bijl D, Bleichrodt RP, Hansson B, Voss A. Surgical smoke and infection control. J Hosp Infect. 2006; 62:1-5.

5. Kwak HD, Kim SH, Seo YS, et al. Detecting hepatitis B virus in surgical smoke emitted during laparoscopic surgery. Occup Environ Med. 2016; 73:857-863.

6. Choi SH, Kwon TG, Chung SK, Kim TH. Surgical smoke may be a biohazard to surgeons performing laparoscopic surgery. Surg Endosc. 2014; 28:2374-80

7. Repici A, Maselli R, Colombo M, et al. Coronavirus (COVID-19) outbreak: what the department of endoscopy should know. Gastrointest Endosc. 2020; pii: S0016-5107(20)30245-5.

8. Zucco L, Levy N, Ketchandji D, et al. Perioperative Considerations for the 2019 Novel Coronavirus (COVID-19) https://www.apsf.org/ news-updates/perioperative-considerations-for-the-2019-novel-coronavirus-covid-19/. Visited the $15^{\text {th }}$ April 2020.

9. Parsa RS, Dirig NF, Eck IN, Payne III WK. Surgical smoke and the orthopedic implications. The Internet Journal of Orthopedic Surgery 2015; Volume 24 Number 1.

10. Gloster HM Jr, Roenigk RK. Risk of acquiring human papillomavirus from the plume produced by the carbon dioxide laser in the treatment of warts. J Am Acad Dermatol. 1995, 32:436-41.

11. Garden JM, O'Banion MK, Shelnitz LS, et al. Papillomavirus in the vapor of carbon dioxide laser-treated verrucae. JAMA. 1988; 259:1199-1202.

12. Ferenczy A, Bergeron C, Richart RM. Human papillomavirus 
DNA in $\mathrm{CO}_{2}$ laser-generated plume of smoke and its consequences to the surgeon. Obstet Gynecol. 1990; 75:114-118.

13. Baggish MS, Poiesz BJ, Joret D, et al. Presence of human immunodeficiency virus DNA in laser smoke. Lasers Surg Med. $1991 ; 11: 197-203$

14. In SM, Park DY, Sohn IK, et al. Experimental study of the potential hazards of surgical smoke from powered instruments. Br J Surg. 2015; 102:1581-1586.

15. Wisniewski PM, Warhol MJ, Rando RF, et al. Studies on the transmission of viral disease via the $\mathrm{CO}_{2}$ laser plume and ejecta. $J$ Reprod Med. 1990; 35:1117-23.

16. Gu J, Han B, Wang J. COVID-19: Gastrointestinal manifestations and potential fecal-oral transmission. Gastroenterology. March 32020 [Epub ahead of print].

17. Joint GI Society message on COVID-19. Clinical insights for our community of gastroenterologists and gastroenterology care providers. https://www.asge.org/home/joint-gisociety-message-covid-1. Visited the $15^{\text {th }}$ April 2020.

\section{* UrOP Executive Committee}

\section{Giuseppe Ludovico ${ }^{1}$, Angelo Cafarelli ${ }^{2}$, Ottavio De Cobelli ${ }^{3}$, Ferdinando De Marco ${ }^{4}$, Giovanni Ferrari ${ }^{5}$, Stefano Pecoraro ${ }^{6}$, Angelo Porreca ${ }^{7}$, Domenico Tuzzolo ${ }^{8}$}

${ }^{1}$ Department of Urology, "F. Miulli" Hospital, Acquaviva delle Fonti (BA), Italy;

${ }^{2}$ Department of Urology, Villa Igea Clinic, Ancona, Italy;

${ }^{3}$ Department of Urology, European Institute of Oncology, IRCCS - Department of Oncology and Hemato-Oncology,

University of Milan, Milan, Italy;

${ }^{4}$ Department of Urology, "INI" Italian Neurotraumatological Institute, Grottaferrata (Roma), Italy;

${ }^{5}$ Department of Urology, CURE, Modena, Italy;

${ }^{6}$ Department of Urology, Malzoni Clinic Neuromed, Avellino, Italy;

${ }^{7}$ Department of Urology, Policlinico Abano Terme, Abano Terme (PD), Italy;

${ }^{8}$ Department of Urology, "Casa del Sole" Clinic, Formia (LT), Italy.

\section{Correspondence}

Rosario Leonardi, MD (Corresponding Author)

leonardi.r@tiscali.it

Lorenzo Falcone, MD

Vincenzo Grasso, MD

Department of Urology and Andrological Surgery Musumeci GECAS Clinic

Gravina of Catania, Catania (Italy)

Piera Bellinzoni, MD

Luigi Broglia, MD

Davide De Marchi, MD

Guido Giusti, MD

Giovanni Passaretti, MD

Silvia Proietti, MD

Antonio Russo, MD

Giuseppe Saitta, MD

Salvatore Smelzo, MD

Franco Gaboardi, MD

Department of Urology, San Raffaele Turro Hospital, Milan (Italy)

Renzo Colombo, MD

Department of Urology, San Raffaele Hospital, Milan (Italy)

Guglielmo Mantica, MD

guglielmo.mantica@gmail.com

Nazareno Suardi, MD

Department of Urology, IRCCS Policlinico San Martino Hospital,

University of Genova, Genova (Italy) 